\title{
ARE EXTREMELY LUMINOUS FAR-INFRARED GALAXIES THE RESULT OF MERGING QUASAR CORES?
}

\author{
R.P.Norris, Australia Telescope National Facility
}

Extremely Luminous far-infrared galaxies (ELFs) are a class of galaxy discovered independently by several groups. The class is characterised by a quasar-like total luminosity $\left(10^{11}-10^{13} \mathrm{~L}_{\odot}\right)$ which is radiated almost entirely in the far-infrared, and it has been suggested that obscured quasar cores may be responsible for generating this luminosity. Here I demonstrate that ELFs appear in several guises which can be characterised by the number of quasar cores they contain (zero, one or two), and develop a unified model to account for these differences.

\section{ARP 220: EVIDENCE FOR MERGING QUASAR CORES}

Arp 220 (IC 4553) has become regarded by many as the archetypal ELF. Evidence for intense starburst activity in it is provided by the optical spectra and high molecular density (e.g. Solomon et al. 1990). In addition, the disturbed morphology, extended tidal plumes and shock- excited infrared spectrum (Joseph et al. 1984) all strongly suggest that Arp 220 is the result of a recent merger.

However, the 40 or so magnitudes of extinction to the core of Arp 220 prevent us from using optical techniques to probe its innermost secrets. Radio observations, on the other hand, are not affected in this way, and have demonstrated the presence of a quasar-like core. The evidence for this is principally the existence of a compact radio nucleus with a high brightness temperature (Norris, 1985, 1988), but is supported by the broad lines seen in Brackett-alpha emission (DePoy et al. 1987) and in $\mathrm{H} \beta$ absorption (Heckman et al. 1983).

Radio observations at sub-arcsec resolution (Norris, 1988) have produced the surprising result that the nucleus of Arp 220 contains not one but two compact radio cores. These probably represent two separate quasar nuclei for the following reasons: (i) the appearance, surface brightness, and scale of these differ sufficiently from the classical 'radio doubles' to indicate that they cannot be easily explained in terms of lobes from a common nucleus; (ii) VLBI observations (Diamond et al. 1989) indicate that both have pc-sized central components; (iii) high-resolution near-infrared images (Carico et al. 1990; Graham et al. 1990) show two condensations, which are presumably galactic nuclei, coincident with the radio cores.

Each of these cores therefore bears the hallmarks of a compact quasar nucleus, indicating that the pair represents either a gravitationally lensed core or else a pair of merging nuclei. Because of the strong evidence for a merger, and in the absence of any other evidence for a gravitational lens, I consider here only the hypothesis that Arp 220 contains a pair of merging nuclei.

The two nuclei of Arp 220 have a projected separation of about $350 \mathrm{pc}$, compared to the kpc separations normally observed in interacting active galaxies. Modelling and numerical calculations (e.g. Begelman et al. 1980; Roos, 1985) have shown that two merging nuclei would spend only a short period at such a small separation before spiralling 
in towards their common potential well. Thus such a configuration is so rare that it can account for only a small fraction of ELFs.

I conclude that Arp 220 no longer appears archetypal, but instead appears to be an extremely peculiar ELF. It has been noticeable in this conference how many graphs have been shown with their axes extended to accommodate Arp 220! It has many superlative claims: it has the highest far-IR excess of any UGC galaxy; it was the first $\mathrm{OH}$ megamaser (and still the brightest) to be discovered; and it refuses to obey the well- established radioFIR correlation. Its optical spectrum appears at first to be that of a starburst, but closer examination (and infrared spectra) show broad quasar-like wings. Of the tens of ELFs now studied in detail, Arp 220 remains unique. Thus there is no conflict with the inferred brief lifetime and rarity of its closely separated cores.

Arp 220 is in many senses the most extreme ELF, and yet typifies many of the properties of other ELFs, and so we must consider its position in the hierarchy of ELFs.

\section{ARE QUASAR CORES PRESENT IN ALL ELFS?}

The evidence that Arp 220 is powered by an obscured quasar core has led to the suggestion (Sanders et al. 1988) that all such objects are powered by quasar cores, and that ELFs represent nascent quasars. However, the competing hypotheses that at least some of these objects are powered by 'super-starburst' activity or by inter-galactic collisions have also gained currency. The plot is thickened further by the common occurrence of tidal tails, interacting companions, and other evidence of disruption.

A long-baseline radio interferometer provides a keen tool for discriminating between these mechanisms, since such an observation is sensitive only to fine structure with high brightness temperature. The 275-km Parkes-Tidbinbilla Interferometer (PTI) has therefore been used to survey a sample of Seyferts, starbursts, and ELFs. The PTI can detect pcsized quasar cores, with brightness temperatures $\approx 10^{8} \mathrm{~K}$, but is blind to the extended kpc-sized starburst regions which have typical brightness temperatures of $10^{4} \mathrm{~K}$.

In our sample of normal starbursts and Seyferts, we found (Norris et al. 1988, 1990) that radio cores are common in optically selected Seyferts, but rare in optically selected starburst galaxies, confirming that the Seyferts are intrinsically different from starbursts, and verifying the validity of the technique.

When we turned our attention to a sample of ELFs previously classified by their optical spectral line ratios into Seyfert-like and starburst-like, we found that radio cores are common in ELFs with Seyfert-like spectra, but rare otherwise. These results demonstrate that there are two separate populations of objects masquerading as ELFs. In one of these populations, the ELFs do contain quasar cores and betray them by a Seyfert-like spectrum. However, those in the other population, which includes some of the most luminous ELFs, do not, and therefore require some other mechanism to explain their activity.

\section{TOWARDS A UNIFIED MODEL FOR ELFS}

We are forced into the unsatisfactory situation of requiring two different types of ELFs: those with Seyfert-like spectra which often contain a radio core, and those with starburst-like spectra which rarely contain a radio core. Our observations so far place tens of members in each class. Arp 220 appears to occupy a third class: a starburst galaxy 
concealing two quasar cores. Obviously, a model to explain ELFs must explain these results, and must also embody the strong evidence for interactions in ELFs of all classes.

Here I speculate on one such model, although clearly variations on this theme are also possible. I assume that some, but not all, galaxies contain quiescent massive black holes $(\mathrm{MBH})$ at their centres, legacies of their quasar youths, and consider the later stages of the merging of two galaxies. The models and timescales used here are derived from calculations and simulations such as those of Begelman et al. (1980), Roos (1981, 1989), and Hernquist (1989).

(i) Two spiral galaxies, which may or may not contain a quiescent $\mathrm{MBH}$, start the merging process by entering a decaying binary orbit with a separation of a few kpc. During this process, shock waves in the disks of the galaxies cause bursts of star formation and the consequent heating of large masses of dust. In this stage the object appears as a starburst-like ELF. If a MBH is present, then the kinematic disruption within the body of the galaxy may start re-fuelling of the $\mathrm{MBH}$, resulting in a mildly Seyfert-like ELF. The Seyfert or quasar core may not appear optically at this stage because of the high extinction to the galactic centre. This phase lasts for perhaps $10^{9}$ years.

(ii) Dynamical friction eventually causes the two galactic nuclei to approach each other to within a few hundred parsecs, with the luminosity of both starburst and Seyfert activity increasing. If two $\mathrm{MBH}$ are present, both are fuelled, giving the appearance of two independent quasar cores, as in Arp 220. Note that if there are no MBH then no quasar cores will be present, so presumably the object will appear as a particularly luminous starburst-like ELF. This phase lasts for perhaps $10^{7}-10^{8}$ years.

(iii) Eventually, the binary orbit decays and the nuclei merge. The starburst activity and MBH refuelling (if present) will continue, but gradually the dust will be blown away (or converted into stars) exposing the nucleus. The galaxy will then appear as a normal Seyfert or starburst galaxy, depending on whether a $\mathrm{MBH}$ is present. This activity may continue for about $10^{7}-10^{8}$ years until the starburst cycle ends, the MBH loss-cone orbits are depleted, and the galaxy returns once more to its inactive state.

Other embellishments to this model are also possible. For example, we may consider whether we should, instead of simply classifying objects as Seyfert or starburst, place them on a starburst-Liner-Seyfert scale. Their position on this scale would then be determined by the ratio of $\mathrm{MBH}$ to starburst activity, which in turn may depend simply on the masses of $\mathrm{MBH}$ within the galaxies. We can also speculate whether the eventual product of several such mergers might be an elliptical galaxy.

Finally, I note that the model above is not a unique solution to the observational data. It is obvious that there is a diversity of significant relationships between the different classes of active galaxy, and consequently the time is ripe for an 'HR diagram' or 'periodic table' of active galaxies in general. Further observations, particularly at radio and infrared wavelengths, will be required before we can determine whether the model presented here, or some other model, may play a central role in the construction of this diagram.

\section{ACKNOWLEDGEMENTS}

I thank James Graham and Dave Carico for permission to cite material prior to publication, and Dave Allen, Jim Caswell, and Alan Roy for comments on this paper. 


\section{REFERENCES}

Begelman, M. C., Blandford, R. D., \& Rees, M. J., 1980, Nature, 287,307.

Carico, D. P., Graham, J. R., Matthews, K., Wilson, T. D., Soifer, B. T., Neugebauer, G., \& Sanders, D. B., 1990, Astrophys. J., 349, L39.

DePoy, D. L., Becklin, E. E., \& Geballe, T. R., 1987, Astrophys. J. Lett., 316, L63.

Diamond, P. J., Norris, R. P., Baan, W. A., \& Booth, R. S., 1989, Astrophys. J., 340, L49.

Graham, J. R., Carico, D. P., Matthews, K., Neugebauer, G., \& Soifer, B. T., 1990. in preparation.

Heckman, T. M., van Breugel, W., Miley, G. K., \& Butcher, H. R., 1983, Astron. J., $88,1077$.

Hernquist, L., 1989, Nature, 340, 687.

Joseph, R. D., Wright, G. S., \& Wade, R., 1984, Nature, 311, 132.

Norris, R. P., 1985, Mon. Not. R. astr. Soc., 216, 701.

Norris, R. P., 1988, Mon. Not. R. astr. Soc., 230, 345.

Norris, R. P., Kesteven, M. J., Allen, D. A., \& Troup, E. R., 1988, Mon. Not. R. astr. Soc., 234, 51p.

Norris, R. P., Allen, D. A., Sramek, R. A., Kesteven, M. J., \& Troup, E. R., 1990, Astrophys. J., in press.

Roos, N., 1981, Astron. Astrophys., 104, 218.

Roos, N., 1985, Astrophys. J., 294, 479.

Roos, N., 1989, in Active Galactic Nuclei, ed. D. E. Osterbrock \& J. S. Miller, (Kluwer, Dordrecht).

Sanders, D. B., Soifer, B. T., Elias, J. H., Neugebauer, G., and Matthews, K. 1988, Astrophys. J., 328, L35.

Solomon, P. M., Radford, S. J. E, \& Downes, D., 1990, Astrophys. J., 348, L53. 
Kembhavi: Could Sey-Elfs be relativistically beamed Sb-Elfs?

Norris: This is a very interesting question that we are actively pursuing. My quess is that a beaming model (as suggested by Barthel ( 1989 and others may be a factor, but it cannot explain everything. For example, Barthel's model would suggest that we should detect syIs more often than SyIIs, whereas, if anything, we detect more syIIs than SyIs.

Heckman: It is very interesting that you find that many FIRG's/ELF's (like Arp 220) probably have "buried" AGN's. However, it is not clear that these AGN's make important contributions to the overall energy budget. Rieke showed that "ultra-luminous" far-IR galaxies are strongly deficient in hard $X-r a y$ emission compared to the hypothesis that all of the IR emission is powered by a standard QSO. He concluded that AGN's could contribute only a small fraction of the IR luminosity, unless they were very peculiar AGN's that were abnormally weak at $10 \mathrm{keV}$.

Norris: Yes. It's interesting that, although we've shown that some, like Arp 220, do contain a buried quasar, many ELF's don't contain one, and so we are now forced to search for an

alternative mechanism. Given the existence of this mechanism, we must then ask how important it is in things like Arp 220. I don't have an answer, but my quess is that, as you suggest, the quasar core may not at present be the dominant energy source. The existence of a quasar core, however, may still be important as we try to place ELF's into the "periodic table" of galaxies, and the core may evolve with time into a dominant position.

van Breugel: What are the spectral indices of the two radio components in Arp 220? If both components (one is even resolved) have a steep spectral index and indeed are AGN's, then what is the physical nature of the radio emission ( $A G N$ 's at radio wavelengths normally have flat radio spectra and are extremely compact; i.e., synchrotron self-absorbed.

Simkin: Comment on discussion by Norris and Van Breuge1--"Why is the core of Arp 220 not a flat spectrum radio source?" (by Van Breugel). Seyferts are not radio loud.

Norris: I agree. It's dangerous to assume that seyferts should have flat-spectrum cores like the radio-loud quasars do.

Observationally, most seyferts do not seem to have flat-spectrum cores, and their radio luminosities would classify most of them as radio-quiet quasars. 
Norris: They both have steep $(\alpha \sim-0.7)$ indices, but then observationally I would say that most seyferts have steepspectrum cores, too. As for the physical origin of this spectral index, I'm not sure. what it's telling us. Perhaps an appreciable amount of the core emission is actually from a nuclear starburst. In the case of Arp $220 \mathrm{I}$ presume that, in addition to quasar cores, starburst activity contributes a substantial fraction of the energy. 